Relations industrielles

Industrial Relations

\title{
Work Hazards and Industrial Conflicts, by Carl Gersuny, University Press of New England, Hanover, N.H., 1981, 162 pp., Lilb. of Congress Catalog Card No 80-51506.
}

\section{René Boulard}

Volume 38, numéro 3, 1983

URI : https://id.erudit.org/iderudit/029393ar

DOI : https://doi.org/10.7202/029393ar

Aller au sommaire du numéro

Éditeur(s)

Département des relations industrielles de l'Université Laval

ISSN

0034-379X (imprimé)

1703-8138 (numérique)

Découvrir la revue

Citer ce compte rendu

Boulard, R. (1983). Compte rendu de [Work Hazards and Industrial Conflicts, by Carl Gersuny, University Press of New England, Hanover, N.H., 1981, 162 pp., Lib. of Congress Catalog Card No 80-51506.] Relations industrielles / Industrial Relations, 38(3), 686-686. https://doi.org/10.7202/029393ar

Tous droits réservés @ C Département des relations industrielles de l'Université Laval, 1983
Ce document est protégé par la loi sur le droit d'auteur. L'utilisation des services d'Érudit (y compris la reproduction) est assujettie à sa politique d'utilisation que vous pouvez consulter en ligne.

https://apropos.erudit.org/fr/usagers/politique-dutilisation/ 
general socio-economic situation of the country plays a major role in this respect.

The focus on conflict may lead in sociological research to the neglect of the phenomenon of co-operation and this study has suffered to some extent in this respect. The factories selected for this study appear to be relatively 'peaceful' and the assumption that some elements of conflict have to found in them seems to be a little risky. In the subtile of the book the word 'resistance' was included but in the content not much justification for it can be located. Probably the authors would gain more by selecting for their study the much clearer an convincing cases of conflict-ridden factories.

\section{Alexander J. MATEJKO}

University of Alberta

\section{Work Hazards and Industrial Conflict, by Carl Gersuny, University Press of New England, Hanover, N.H., 1981, 162 pp., Lib. of Congress Catalog Card No 80-51506}

Ce volume, à saveur et contenu exclusivement américains, tente de situer le problème de la santé et de la sécurité du travail dans le contexte plus large du conflit industriel, voire même de la lutte des classes. Le premier chapitre intitulé A Part of the Class Struggle pose la problématique de base qui sert à l'analyse de tout le volume. Les chapitres 2 à 5 décrivent et analysent le problème des dangers du travail aux États-Unis et particulièrement dans les filiatures de la Nouvelle Angleterre au début du siècle. Le chapitre 2 décrit le climat des relations du travail à cette époque aux États-Unis. Les chapitres 3 et 4 analysent le rôle des parties et des tiers (médecins, avocats, détectives privés, etc.) dans un certain nombre de dossiers d'accidents et de maladies industrielles. Le chapitre 5 présente brièvement l'évolution de la situation de 1910 à
1970 et le dernier chapitre tente de faire le point sur la situation actuelle "continuity and change», suite au Workmen's Compensation Act et surtout à l'Occupational Safety and Health Act.

Cet ouvrage n'a que 162 pages. Les chapitres 2 à 5 en occupent près de 100. Mais leur lecture est tellement pénible, fastidieuse et non-pertinente qu'on ne voit pas le jour d'en sortir. L'argument principal n'est pas dénué de fondement et pourrait prêter à une analyse intéressante: dans les relations du travail, la santé et la sécurité des travailleurs représentent un objectif secondaire par rapport aux profits de l'employeur. Elles ont donc toujours été sacrifiées et monnayées à l'avantage de ce dernier. Mais pourquoi se cantonner à la Nouvelle-Angleterre, dans les filiatures, avec des études de cas dont la plus récente a connu son dénouement en 1912? Le volume présente sans doute un intérêt historique certain. Mais pour celui qui est intéressé aux relations industrielles, à la santé et à la sécurité du travail, les pièces versées au dossier et apportées en preuve dégagent une forte odeur de boule à mites ancestrale.

Les deux derniers chapitres tentent de rattrapper le morceau, mais il est trop tard. L'auteur finit par admettre que les pires abus du tournant du siècle (au moment où le bras d'un garçon de 14 ans valait $\$ 4.60$, p. 55 ) sont pratiquement impossibles suite à la montée du syndicalisme et aux modifications apportées aux lois. Mais comme ceci risque de perturber trop sérieusement la preuve historique développée par l'auteur, il n'insiste pas outre mesure.

Bref, un volume dont les chapitres 1 et 6 méritent d'être lus et les chapitres 2 à 5 peuvent être examinés rapidement, à moins qu'on ne se complaise dans un «musée des horreurs».

René BOULARD

Université Laval 